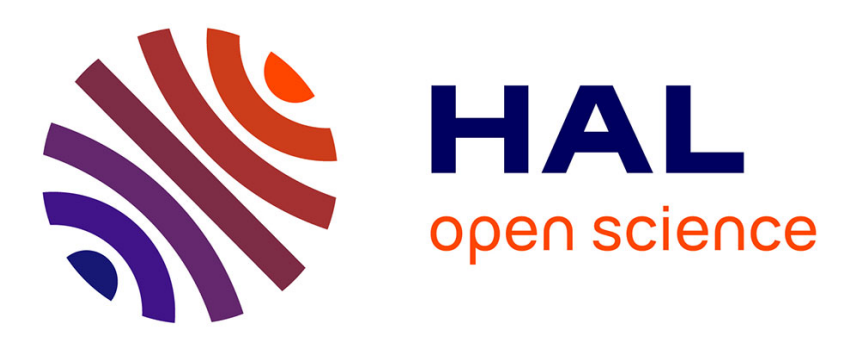

\title{
Etude in situ par EXAFS de la réaction de reformage sur les systèmes $\mathrm{Pt} / \mathrm{Al} 2 \mathrm{O} 3$ et $\mathrm{PtRe} / \mathrm{Al} 2 \mathrm{O} 3$ sulfurés
}

\author{
A. Bensaddik, Dominique Bazin, H. Dexpert, A. Caballero, J. Lynch, B.
} Didillon

\section{- To cite this version:}

A. Bensaddik, Dominique Bazin, H. Dexpert, A. Caballero, J. Lynch, et al.. Etude in situ par EXAFS de la réaction de reformage sur les systèmes $\mathrm{Pt} / \mathrm{Al} 2 \mathrm{O} 3$ et $\mathrm{PtRe} / \mathrm{Al} 2 \mathrm{O} 3$ sulfurés. Journal de Physique IV Proceedings, 1996, 06 (C4), pp.C4-493-C4-500. 10.1051/jp4:1996447 . jpa-00254330

\section{HAL Id: jpa-00254330 https://hal.science/jpa-00254330}

Submitted on 1 Jan 1996

HAL is a multi-disciplinary open access archive for the deposit and dissemination of scientific research documents, whether they are published or not. The documents may come from teaching and research institutions in France or abroad, or from public or private research centers.
L'archive ouverte pluridisciplinaire HAL, est destinée au dépôt et à la diffusion de documents scientifiques de niveau recherche, publiés ou non, émanant des établissements d'enseignement et de recherche français ou étrangers, des laboratoires publics ou privés. 


\title{
Etude in situ par EXAFS de la réaction de reformage sur les systèmes $\mathrm{Pt} / \mathrm{Al}_{2} \mathrm{O}_{3}$ et $\mathrm{PtRe} / \mathrm{Al}_{2} \mathrm{O}_{3}$ sulfurés
}

\author{
A. Bensaddik, D. Bazin, H. Dexpert, A. Caballero*, J. Lynch** et B. Didillon** \\ LURE, Bât. 209D, Université Paris-Sud, 91405 Orsay cedex, France \\ * ICMS, P.O. Box 1115, 41080 Sevilla, Spain \\ ** Institut Français du Pétrole, BP. 311, 92506 Rueil-Malmaison cedex, France
}

\begin{abstract}
Résumé: La technique EXAFS a été utilisée pour suivre in situ dans les conditions de reformage $\left(\mathrm{T}=500^{\circ} \mathrm{C}\right.$, $\mathrm{P}\left(\mathrm{H}_{2}\right)=8$ bars, $\mathrm{H}_{2}: \mathrm{n}_{-} \mathrm{C}_{7} \mathrm{H}_{16}=1: 1$ et $\left.\mathrm{T}=300^{\circ} \mathrm{C}, \mathrm{P}_{2} \mathrm{H}_{2}\right)=3$ bars $\mathrm{H}_{2}: \mathrm{n}_{-}-\mathrm{C}_{7} \mathrm{H}_{16}=1: 1$ ), l'évolution de la structure des deux catalyseurs $\mathrm{Pt} / \mathrm{Al}_{2} \mathrm{O}_{3}-\mathrm{Cl}$ et $\mathrm{PtRe} / \mathrm{Al}_{2} \mathrm{O}_{3}-\mathrm{Cl}$ sulfurés. On a observé la grande stabilité de la phase métallique du bimétallique comparée à celle du monométallique. La phase métallique de ce dernier subit une agglomération dès la réduction à $500^{\circ} \mathrm{C}$, processus qui s'accentue jusqu'à la fin de la réaction de reformage.

Abstract: EXAFS has been used to follow in situ the structural evolution of sulfided $\mathrm{Pt} / \mathrm{Al}_{2} \mathrm{O}_{3}-\mathrm{Cl}_{\text {and }}$ $\mathrm{PtRe} / \mathrm{Al}_{2} \mathrm{O}_{3}-\mathrm{Cl}$ catalysts during the hydrocarbon reaction $\left(\mathrm{T}=500^{\circ} \mathrm{C}, \mathrm{P}\left(\mathrm{H}_{2}\right)=8\right.$ bars $\mathrm{H}_{2}: \mathrm{n}-\mathrm{C}_{7} \mathrm{H}_{16}=1: 1$ and $\mathrm{T}=300^{\circ} \mathrm{C}, \mathrm{P}\left(\mathrm{H}_{2}\right)=3$ bars, $\mathrm{H}_{2}: \mathrm{n}-\mathrm{C}_{7} \mathrm{H}_{16}=1: 1$ ). The hydrocarbon conversion was monitored, via on line analysis, with a gas chromatograph. For the monometallic, we observe a sintering of the metal particles. In the case of sulfided PtRe/Al $\mathrm{O}_{3}-\mathrm{Cl}$ catalysts no sintering of the metallic particles was observed.
\end{abstract}

\section{INTRODUCTION}

Les catalyseurs métalliques supportés, ceux du reformage notamment, contiennent une quantité très faible de métal. Pour obtenir des informations sur la phase métallique de ces systèmes, la spectroscopie d'absorption $X$ donne de grandes possibilités [1]. L'utilisation de cette technique en caractérisation de ces solides très divisés est en fait devenue d'une grande importance, depuis l'introduction de cellules réactionnelles permettant de suivre in situ l'évolution structurale et électronique de la phase métallique déposée.

La catalyse bifonctionnelle métal-acide par les systèmes $\mathrm{P} / \mathrm{Al}_{2} \mathrm{O}_{3}-\mathrm{Cl}$ et $\mathrm{PtRe} / \mathrm{Al}_{2} \mathrm{O}_{3}-\mathrm{Cl}$ est largement utilisée lors du reformage, processus de l'industrie pétrolière. Gagner en stabilité et parfois en sélectivité est le grand avantage de l'utilisation du catalyseur bimétallique $\mathrm{PtRe} / \mathrm{Al}_{2} \mathrm{O}_{3}-\mathrm{Cl}$ comparé au monométallique $\mathrm{Pt} / \mathrm{Al}_{2} \mathrm{O}_{3}-\mathrm{Cl}$. L'addition du rhénium apporte toutefois un effet indésirable en raison de son grand pouvoir hydrogénolysant. Afin de réduire cet effet, le système platine-rhénium est présulfuré avant son utilisation industrielle [2,3].

Dans le but de comprendre le rôle du second métal (rhénium), une étude in situ par EXAFS a été menée précédemment sur la sulfuration du monométallique $\mathrm{P} / \mathrm{Al}_{2} \mathrm{O}_{3}-\mathrm{Cl}$ et du bimétallique $\mathrm{PtRe} / \mathrm{Al}_{2} \mathrm{O}_{3}-\mathrm{Cl}$ [4]. Les informations structurales directes obtenues nous ont permis de montrer clairement la différence de comportement de ces deux systèmes catalytiques vis a vis de cette sulfuration. Ainsi dans le but de completer ce travail, on a réalisé l'étude in situ par EXAFS de la réaction de reformage sur les systèmes $\mathrm{Pt} / \mathrm{Al}_{2} \mathrm{O}_{3}-\mathrm{Cl}$ et $\mathrm{PtRe} / \mathrm{Al}_{2} \mathrm{O}_{3} \mathrm{Cl}$ sulfurés. Nous avons recherché des informations structurales directes en conditions réactionnelles. Le but est d'établir au mieux le lien entre les changements qui interviennent dans la structure de notre catalyseur et son activité catalytique, puisque nous collectons par chromatographie en ligne les produits issus de la réaction. Cette recherche nous permet donc une comparaison cristallochimique directe entre les comportements du monométallique et du bimétallique sulfurés dans les conditions réactionnelles de reformage. 


\section{EXPERIENCE}

Le monométallique $\mathrm{Pt} / \mathrm{Al}_{2} \mathrm{O}_{3}$ chloré est préparé par imprégnation d'une alumine gamma avec une solution de l'acide hexachloroplatinique $\left(\mathrm{H}_{2} \mathrm{PtCl}_{6}\right)$. L'alumine imprégnée est séchée à $110^{\circ} \mathrm{C}$ pendant une douzaine d'heures puis calcinée dúrant deux heures sous air sec à $500^{\circ} \mathrm{C}$. La préparation du catalyseur bimétallique $\mathrm{PtRe} / \mathrm{Al}_{2} \mathrm{O}_{3}$ est réalisée selon le principe précédant mais par imprégnation successive, en fixant d'abord le platine comme dans le cas du monométallique. La fixation du rhénium est effectúe par ajout d'une solution aqueuse de la phase du précurseur $\mathrm{ReO}_{4} \mathrm{NH}_{4}$, en déterminant la concentration de cette solution en fonction de la teneur en rhénium recherchée [5].

Les catalyseurs étudiés dans ce travail sont donc sous forme de poudre et l'analyse chimique préalable montre que le monométallique $\mathrm{Pt} / \mathrm{Al}_{2} \mathrm{O}_{3}$ chloré contient $1 \%$ en poids de platine et $1,2 \%$ en poids de chlore. Pour le bimétallique $\mathrm{PtRe} / \mathrm{Al}_{2} \mathrm{O}_{3}$ chloré, la teneur en métal est de $1 \%$ en poids de platine, $1 \%$ en poids de rhénium et toujours $1,2 \%$ en poids de chlore. Des mesures classiques d'adsorption permettent d'évaluer la surface spécifique à $200 \mathrm{~m}^{2} \mathrm{~g}^{-1}$ aussi bien pour les échantillons monométalliques que bimétalliques. La similarité de ces valeurs, surface spécifique, teneur en éléments d'intérêt, a été choisie pour pouvoir comparer avec le moins de variables possibles le comportement de ces deux systèmes. Les pourcentages en poids sont très proches des compositions industriellement utilisées pour placer les résultats de ce travail dans un contexte direct d'application, du moins d'extrapolation facile, dans le milieu réel d'une unité de reformage.

Les mesures EXAFS sont réalisées au centre synchrotron de LURE à Orsay, en utilisant le rayonnement électromagnétique issu de l'anneau de stockage DCl (Dispositif de Collision dans l'Igloo). Cet anneau est caractérisé par une énergie de $1,85 \mathrm{GeV}$ et un courant machine de l'ordre de $300 \mathrm{~mA}$. Les spectres EXAFS sont enregistrés au seuil $\mathrm{LII}_{\mathrm{II}}$ du platine (11564 $\mathrm{eV})$ pour le monométallique et aux seuils LIII du platine et du rhénium (10534 eV) pour le bimétallique sur le dispositif expérimental "ExafsIV-D44". Le faisceau "blanc" polychromatique est monochromatisé par une double réflexion de Bragg sur des cristaux de silicium (Si111). Les mesures d'absorption de rayons X sont réalisées par transmission, on a utilisé comme système de détection deux chambres à ionisation pour mesurer l'intensité du faisceau avant et après traversée de l'échantillon par les photons $\mathrm{X}$.

Le catalyseur à étudier est sous forme de poudre finement broyée. La taille des grains est comprise entre 100 et $200 \mu \mathrm{m}$ afin de permettre une meilleure circulation des gaz. L'échantillon est placé dans un réacteur haute température, haute pression qui permet de suivre son évolution structurale au cours de la réduction sous dihydrogène et au cours du cokage en présence d'hydrocarbures. Cette cellule est couplée à un chromatographe en phase gazeuse pour l'analyse simultanee des produits issus de la transformation catalytique des hydrocarbures pendant la réaction de cokage. Cet ensemble réactionnel permet de travailler dans un domaine de température compris entre $20^{\circ} \mathrm{C}<\mathrm{T}<500^{\circ} \mathrm{C}$, le débit d'hydrogène pouvant varier de 0 a 5 litres par heure avec une pression de 1 a 15 bars. Le volume d'hydrocarbures pouvant être injecté peut varier de 0 à $100 \mathrm{~cm}^{3 / h}$ avec une pression de 1 à 15 bars.. L'ensemble est installé sur le trajet du faisceau de rayons $\mathrm{X}$, entre les deux chambres de détection.

Une fois le catalyseur placé dans la cellule réactionnelle, on realise une réaction de reformage du n-heptane selon un protocole expérimental qui comprend trois étapes :

- étape 1 : réduction sous hydrogène à $500^{\circ} \mathrm{C}$ pendant $2 \mathrm{~h} 40 \mathrm{mn}$ pour le bimétallique $\mathrm{PtRe} / \mathrm{Al}_{2} \mathrm{O}_{3}-\mathrm{Cl}$ sulfuré et $3 \mathrm{~h} 30 \mathrm{mn}$ pour le monométallique $\mathrm{P} / \mathrm{Al}_{2} \mathrm{O}_{3}-\mathrm{Cl}$ sulfuré;

- étape II : réaction de reformage à la température de $500^{\circ} \mathrm{C}$, une pression d'hydrogène de 8 bars et un rapport hydrogène/hydrocarbure de l'ordre de 1. Le temps de réaction dans ces conditions pour le catalyseur $\mathrm{Pt} / \mathrm{Al}_{2} \mathrm{O}_{3}-\mathrm{Cl}$ est de $4 \mathrm{~h} 20 \mathrm{mn}$ et de 6 heures pour le bimétallique $\mathrm{PtRe} / \mathrm{Al}_{2} \mathrm{O}_{3}-\mathrm{Cl}$;

- étape III : changement in situ des conditions de la réaction de reformage, toujours avec le même rapport hydrogène/hydrocarbure. On réalise alors un palier à $300^{\circ} \mathrm{C}$ avec une pression dhydrogène de 3 bars. Cette étape dure 2 heures pour le bimétallique et 1 h50mn pour le monométallique. Le temps global d'injection d'hydrocarbures (étape I plus étape II) est de 8 heures pour le bimétallique et de $6 \mathrm{hlO}$ mn pour le monométallique. Cette différence de temps est due essentiellement au fait que pour le bimétallique l'enregistrement se fait aux deux seuils d'absorption X (LIII du platine et du rhénium). 
Dans les conditions normales d'utilisation industrielle, ces catalyseurs presentent une durée de vie très importante et les premiers signes de désactivation n'apparaissent qu'après plusieurs dizaines d'heures de fonctionnement. La limitation dans le temps d'une experience EXAFS nous amène à choisir, pour notre réaction de reformage, un rapport hydrogène/hydrocarbure relativement faible, de l'ordre de 1, qui favorise la désactivation rapide du catalyseur. Ceci nous permet de rendre compte des changements structuraux survenus lors de cette désactivation de manière réaliste par rapport à la véritable réaction, tout en restant dans les limites de temps de collecte de données raisonnables (de l'ordre de 48 heures d'affilée). Ces contraintes nous imposent également de relever les spectres aux températures de réaction.

\section{CONDITIONS D'ANALYSE EXAFS}

Pour notre travail, cinq références expérimentales ont été utilisées dont nous précisons pour chacune les caractéristiques structurales (coordinences et distances interatomiques) : l'oxyde de platine $\beta-P t O_{2}(N=6, R=2.04 \AA)$ pour les liaisons platine-oxygène, la feuille de platine $(\mathrm{N}=12, \mathrm{R}=2.77 \AA)$ pour les liaisons platine-platine, une solution d'acide hexachloroplatinique $\left(\mathrm{H}_{2} \mathrm{PlCl}_{6}\right),(\mathrm{N}=6, \mathrm{R}=2.32 \AA)$ pour les liaisons platine-chlore, platine-soufre, thénium-chlore et rhénium-soufre, un catalyseur réduit $15 \% \mathrm{Re}^{-} \mathrm{Al}_{2} \mathrm{O}_{3}(\mathrm{~N}=12, \mathrm{R}=2.77 \AA)$ pour les liaisons rhénium-rhénium, une solution de perrhénate de rhénium $\left(\mathrm{NH}_{4} \mathrm{ReO}_{4}\right),(\mathrm{N}=4,1.77 \AA)$ pour les liaisons rhénium-oxygène.

L'exploitation des spectres EXAFS collectés en conditions réactionnelles présente certaines difficultés. En effet, l'environnement du catalyseur formé par plusieurs éléments permettant le travail in situ réduit considérablement le nombre de photons utiles et de plus, nos systèmes catalytiques étudiés dans ce travail sont très peu chargés ( $1 \% \mathrm{Ptl} \% \mathrm{Re} / \mathrm{Al}_{2} \mathrm{O}_{3}$ et $1 \% \mathrm{Pt} / \mathrm{Al}_{2} \mathrm{O}_{3}$ ). De ce fait l'analyse quantitative n'est réalisable que pour la première sphère de coordination de l'élément photoexcité (le platine pour le monométallique $\mathrm{Pt} / \mathrm{Al}_{2} \mathrm{O}_{3}$, platine et rhénium pour le bimétallique PtRe/Al $\mathrm{O}_{3}$ ) et le rapport signal sur bruit très faible vers les grandes distances rend l'analyse des troisièmes et quatrièmes voisins non réalisable.

Les analyses quantitatives, présentées par la suite, sont réalisées en utilisant au maximum deux couches. Pour le catalyseur bimétallique, l'analyse structurale par EXAFS ne peut séparer que très difficilement les contributions associées au platine de celles relatives au rhénium ( $Z=78$ et 75 respectivement). Ainsi, nous appellerons métal $M$, sans les séparer, la contribution rétrodiffusante des voisins platine ou rhénium sur l'un ou l'autre de ces éléments. La transformée de Fourier de l'oscillation EXAFS est obtenue en utilisant une fenêtre de Hanning entre 40-500 eV. Par une transformée de Fourier inverse, avec un filtre entre 1.0-3.5 $\AA$, on obtient la modulation EXAFS filtrée relative à la couche qu'on veut analyser. La détermination des paramètres structuraux (le nombre de voisin et la distance interatomique) se fait par l'utilisation des fonctions phase et amplitude des différents composés de références, dont les structures cristallographiques connues et très voisines de celles suspectées exister dans l'échantillon lors de l'enregistrement du spectre EXAFS. Tous les affinements sont réalisés dans l'espace des énergies (E) et l'espace des distances (R). Un exemple de la bonne qualité des simulations entre nos spectres expérimentaux et les références est donné figure 1.
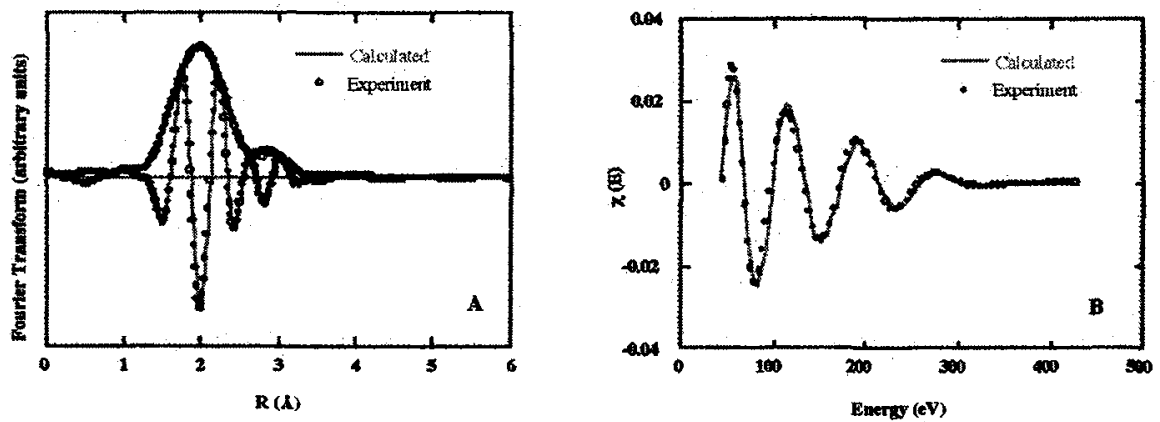

Figure 1: Simulation obtenue au seuil $\mathrm{LIII}$ du platine pour le catalyseur $\mathrm{Pt} / \mathrm{Al}_{2} \mathrm{O}_{3}-\mathrm{Cl}$. La figure $1 \mathrm{~A}$ présente la simulation dans l'espace des distances $(R)$ et la figure $1 B$ celle dans l'espace des énergies $(E)$.

Simulation obtained at the LII edge of platinum for the monometallic $\mathrm{Pt} / \mathrm{Al}_{2} \mathrm{O}_{3}-\mathrm{Cl}$. The fit was performed in $\mathrm{R}$ space (figure $1 \mathrm{~A}$ ) and $\mathrm{E}$ space (figure 1B). 


\section{RESULTATS EXPERIMENTAUX}

\subsection{La désactivation du monométallique $1 \% \mathrm{Pt} / \mathrm{Al}_{2} \mathrm{O}_{3}-\mathrm{Cl}$ sulfuré}

Les analyses numériques EXAFS sont effectuées à différentes étapes du traitement. Les résultats sont regroupés dans le tableau 1.

Tableau 1: Catalyseur PU/Al2 $\mathrm{O}_{3}-\mathrm{Cl}$ sulfuré: paramètres structuraux au seuil LIII du platine EXAFS parameters (LII platinum edge) for sulfided $\mathrm{P} v \mathrm{Al}_{2} \mathrm{O}_{3}-\mathrm{Cl}$ catalyst.

\begin{tabular}{|c|c|c|c|}
\hline $\mathrm{Pt} / \mathrm{Al}_{2} \mathrm{O}_{3}-\mathrm{Cl}$ & \multicolumn{3}{|c|}{$\begin{array}{l}\text { Paramètres EXAFS obtenus au seuil LiI du platine } \\
\text { EXAFS parameters obtained at the } L_{\text {III }} \text { edge of platinum }\end{array}$} \\
\hline $\begin{array}{l}\text { Catalyseur initial } \\
\text { Initial state }\end{array}$ & $\begin{array}{l}\text { NPtS }=2.7 \\
\text { NPtPt }=1.7\end{array}$ & $\begin{aligned} \text { RPtS } & =2.32 \AA \\
\text { RPtPt } & =2.69 \AA\end{aligned}$ & $\begin{array}{l}\Delta \mathrm{OPtS}=0.07 \AA \\
\triangle \mathrm{OPtPt}=0.02 \AA\end{array}$ \\
\hline \multicolumn{4}{|c|}{$\begin{array}{l}\text { Réduction sous hydrogene d } 500^{\circ} \mathrm{C} \\
\text { First reduction under hydrogen at } 500^{\circ} \mathrm{C}\end{array}$} \\
\hline $\begin{array}{l}\text { Après } 2 \mathrm{~h} 20 \mathrm{mn} \\
\text { After } 2 \mathrm{~h} 20 \mathrm{mn}\end{array}$ & $\begin{aligned} \mathrm{NPtS} & =0.8 \\
\mathrm{NPtPt} & =6.0\end{aligned}$ & $\begin{array}{l}\text { RPtS }=2.28 \AA \\
\text { RPtPt }=2.68 \AA\end{array}$ & $\begin{array}{l}\triangle O P t S=0.08 \AA \\
\triangle O P t P t=0.05 \AA\end{array}$ \\
\hline $\begin{array}{l}\text { Apres } 3 \mathrm{~h} 15 \mathrm{mn} \\
\text { After } 3 \mathrm{~h} 15 \mathrm{mn}\end{array}$ & $\begin{aligned} \text { NPtS } & =0.6 \\
N P_{t P t} & =6.0\end{aligned}$ & $\begin{array}{l}\text { RPtS }=2.30 \AA \\
\operatorname{RPtPt}=2.67 \AA\end{array}$ & $\begin{aligned} \Delta \mathrm{OP}_{\mathrm{tS}} & =0.07 \AA \\
\Delta \mathrm{OP} \mathrm{Pt} & =0.04 \AA\end{aligned}$ \\
\hline \multicolumn{4}{|c|}{$\begin{array}{l}\text { Première réaction de reformage }\left(\mathrm{T}=500^{\circ} \mathrm{C}, \mathrm{P}\left(\mathrm{H}_{2}\right)=8 \text { bars }\right) \\
\text { First reforming reaction }\left(\mathrm{T}=500^{\circ} \mathrm{C}, \mathrm{P}\left(\mathrm{H}_{2}\right)=8 \text { bars }\right)\end{array}$} \\
\hline $\begin{array}{l}\text { Apres } 1 \mathrm{~h} 10 \mathrm{mn} \\
\text { After } 1 \mathrm{~h} 10 \mathrm{mn}\end{array}$ & $\begin{array}{l}\text { NPtS }=0.6 \\
\text { NPtPt }=7.0\end{array}$ & 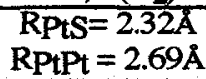 & $\begin{array}{l}\Delta O P t S=0.09 \AA \\
\Delta O P t P t=0.04 \AA\end{array}$ \\
\hline $\begin{array}{l}\text { Après } 2 \mathrm{~h} 30 \mathrm{mn} \\
\text { After } 2 \mathrm{~h} 30 \mathrm{mn}\end{array}$ & $\begin{aligned} \text { NPtS } & =0.4 \\
\text { NPtPt } & =7.0\end{aligned}$ & $\begin{array}{r}\mathrm{RPtS}=2.33 \AA \\
\mathrm{RPtPt}=2.67 \AA\end{array}$ & $\begin{array}{l}\Delta O \mathrm{PtS}_{\mathrm{S}}=0.09 \AA \\
\triangle \mathrm{OPtPt}=0.05 \AA\end{array}$ \\
\hline $\begin{array}{l}\text { Après } 4 \mathrm{hoOmn} \\
\text { After } 4 \mathrm{~h} 00 \mathrm{mn}\end{array}$ & $\begin{array}{l}\text { NPtS }=0.5 \\
\text { NPtPt }=7.0\end{array}$ & 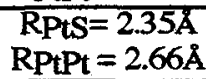 & $\begin{array}{l}\Delta \mathrm{OPtS}=0.07 \AA \\
\Delta \mathrm{OPtPt}=0.05 \AA\end{array}$ \\
\hline \multicolumn{4}{|c|}{$\begin{array}{l}\text { Seconde réaction de reformage }\left(\mathrm{T}=300^{\circ} \mathrm{C}, \mathrm{P}\left(\mathrm{H}_{2}\right)=3 \text { bars }\right) \\
\text { Second reforming reaction }\left(\mathrm{T}=300^{\circ} \mathrm{C}, \mathrm{P}\left(\mathrm{H}_{2}\right)=3 \text { bars }\right)\end{array}$} \\
\hline $\begin{array}{l}\text { Après } 1 \mathrm{~h} 50 \mathrm{mn} \\
\text { After } 1 \mathrm{~h} 50 \mathrm{mn}\end{array}$ & $\begin{aligned} \mathrm{NPtS} & =0.4 \\
\mathrm{NPtPt} & =7.3\end{aligned}$ & $\begin{aligned} \mathrm{RPtS} & =2.33 \AA \\
\mathrm{RPtPt} & =2.66 \AA\end{aligned}$ & $\begin{array}{l}\Delta \mathrm{OPtS}=0.08 \AA \\
\Delta \mathrm{OPtPt}=0.01 \AA\end{array}$ \\
\hline $\begin{array}{l}\text { Fin de la réaction } \\
\text { End of treatment }\end{array}$ & $\begin{aligned} \mathrm{NPtS} & =0.4 \\
\mathrm{NPtPt} & =7.8\end{aligned}$ & $\begin{array}{r}\text { RPtS }=2.32 \AA \\
\text { RPtPt }=2.69 \AA\end{array}$ & $\begin{array}{l}\Delta \mathrm{OPtS}=0.04 \AA \\
\Delta \mathrm{OPtPt}=0.03 \AA\end{array}$ \\
\hline
\end{tabular}

Cette série de mesures nous permet d'observer que le soufre adsorbe sur la phase métallique du catalyseur $1 \% \mathrm{Pt} / \mathrm{Al}_{2} \mathrm{O}_{3}-\mathrm{Cl}$ n'est plus stable à $500^{\circ} \mathrm{C}$. L'augmentation du nombre de voisins platine, après la réduction à $500^{\circ} \mathrm{C}$, indique une agglomération de la phase métallique. Elle s'accentue jusqu'à la fin de la réaction de reformage, le nombre de voisins platine passant de 6 après réduction à 7.8 en fin de réaction de reformage.

\section{4..2 La désactivation du bimétallique $1 \% \mathrm{Pt}-1 \% \mathrm{Re} / \mathrm{Al}_{2} \mathrm{O}_{3}-\mathrm{Cl}$ sulfuré}

Les analyses numériques EXAFS (seuil Lm du platine : tableau 2, seuil $\mathrm{Lm}$ du rhénium: tableau 3) nous permettent de suivre l'évolution de l'environnement du platine et du rhénium à différentes étapes du traitement. Tout d'abord, l'ensemble des résultats indique une adsorption préférentielle du soufre sur le hiénium ( $\mathrm{N}_{\mathrm{PtS}}=1.0, \mathrm{~N}_{\mathrm{ReS}}=2.0$ ), résultat que nous avons déjà eu l'occasion de commenter. De plus, les liaisons platine-métal formées s'avèrent plus stables par rapport à celles présentes dans le monométallique. 
Tableau 2: Catalyseur PtRe/AbO3-Cl sulfure: paramètres structuraux an seuil LIII du platine EXAFS parameters (LII platinum edge) for sulfided $\mathrm{PtRe} / \mathrm{Al}_{2} \mathrm{O}_{3}-\mathrm{Cl}$ catalyst

\begin{tabular}{|c|c|c|c|}
\hline $\mathrm{PtRe} / \mathrm{Al}_{2} \mathrm{O}_{3}-\mathrm{CI}$ & \multicolumn{3}{|c|}{$\begin{array}{l}\text { Parametres EXAFS obtenus au seuil Lm du platine } \\
\text { EXAFS parameters obtained at the LIII edge of platinum }\end{array}$} \\
\hline $\begin{array}{l}\text { Catalyseur initial } \\
\text { Initial state }\end{array}$ & $\begin{aligned} \mathrm{NPtS} & =1.0 \\
\mathrm{NPtM} & =3.9\end{aligned}$ & $\begin{array}{r}\text { RPtS }=2.31 \AA \\
\text { RPtM }=2.71 \AA\end{array}$ & $\begin{aligned} \triangle O P t S & =0.00 \AA \\
\triangle O P t M & =0.04 \AA\end{aligned}$ \\
\hline \multicolumn{4}{|c|}{$\begin{array}{l}\text { Réduction sous hydrogène a } 500^{\circ} \mathrm{C} \\
\text { Reduction under hydrogen at } 500^{\circ} \mathrm{C}\end{array}$} \\
\hline $\begin{array}{l}\text { Après } 2 \mathrm{~h} 30 \mathrm{mn} \\
\text { After } 2 \mathrm{~h} 30 \mathrm{mn}\end{array}$ & $\begin{aligned} \text { NPtS } & =0.9 \\
\text { NPtM } & =4.3\end{aligned}$ & $\begin{array}{l}\text { RPtS }=2.36 \AA \\
\text { RPtM }=2.69 \AA\end{array}$ & $\begin{aligned} \Delta \mathrm{OPtS} & =0.06 \AA \\
\Delta \mathrm{OPtM} & =0.02 \AA\end{aligned}$ \\
\hline \multicolumn{4}{|c|}{$\begin{array}{l}\text { Première réaction de reformage }\left(\mathrm{T}=500^{\circ} \mathrm{C}, \mathrm{P}\left(\mathrm{H}_{2}\right)=8 \text { bars }\right) \\
\text { First reforming reaction }\left(\mathrm{T}=500^{\circ} \mathrm{C}, \mathrm{P}\left(\mathrm{H}_{2}\right)=8 \mathrm{bars}\right)\end{array}$} \\
\hline $\begin{array}{l}\text { Après } 1 \mathrm{~h} 25 \mathrm{mn} \\
\text { After } 1 \mathrm{~h} 25 \mathrm{mn}\end{array}$ & $\begin{array}{l}\text { NPtS }=0.85 \\
\text { NPtM }=4.4\end{array}$ & $\begin{aligned} \text { RPtS } & =2.33 \AA \\
\text { RPtM } & =2.72 \AA\end{aligned}$ & $\begin{aligned} \Delta \sigma_{\mathrm{PtS}} & =0.07 \AA \\
\Delta \mathrm{OPtM} & =0.02 \AA\end{aligned}$ \\
\hline $\begin{array}{l}\text { Après } 2 \mathrm{~h} \\
\text { After } 2 \mathrm{~h}\end{array}$ & $\begin{array}{l}\text { NPtS }=0.8 \\
\text { NPtM }=4.0\end{array}$ & $\begin{array}{l}\text { RPtS }=2.31 \AA \\
\text { RPtM }=2.73 \AA\end{array}$ & $\begin{aligned} \Delta \mathrm{OPtS} & =0.05 \AA \\
\Delta \mathrm{OPtM} & =0.00 \AA\end{aligned}$ \\
\hline $\begin{array}{l}\text { Apres } 5 \mathrm{~h} \\
\text { After } 5 \mathrm{~h}\end{array}$ & $\begin{array}{l}\text { NPtS }=0.8 \\
\text { NPtM }=3.9\end{array}$ & $\begin{array}{l}\mathrm{RPtS}=2.31 \AA \\
\mathrm{RPtM}=2.71 \AA\end{array}$ & $\begin{array}{l}\Delta O \mathrm{PtS}_{\mathrm{P}}=0.07 \AA \\
\Delta \mathrm{OPtM}=0.02 \AA\end{array}$ \\
\hline \multicolumn{4}{|c|}{$\begin{array}{l}\text { Seconde réaction de reformage }\left(\mathrm{T}=300^{\circ} \mathrm{C}, \mathrm{P}\left(\mathrm{H}_{2}\right)=3 \text { bars }\right) \\
\text { Second reforming reaction }\left(\mathrm{T}=300^{\circ} \mathrm{C}, \mathrm{P}\left(\mathrm{H}_{2}\right)=3 \text { bars }\right)\end{array}$} \\
\hline $\begin{array}{l}\text { Après } 45 \mathrm{mn} \\
\text { After } 45 \mathrm{mn}\end{array}$ & $\begin{array}{l}\text { NPtS }=0.8 \\
\text { NPtM }=4.0\end{array}$ & $\begin{aligned} \text { RPtS } & =2.30 \AA \\
\text { RPtM } & =2.71 \AA\end{aligned}$ & $\begin{array}{l}\Delta O P t S=0.05 \AA \\
\triangle O P t M=0.03 \AA\end{array}$ \\
\hline $\begin{array}{l}\text { Après } 2 \mathrm{~h} \\
\text { After } 2 \mathrm{~h}\end{array}$ & $\begin{array}{l}\mathrm{NPtS}=0.8 \\
\mathrm{NPtM}=5.2\end{array}$ & $\begin{aligned} \mathrm{RPtS} & =2.34 \AA \\
\mathrm{RPtM} & =2.71 \AA\end{aligned}$ & $\begin{array}{l}\Delta O \mathrm{PtS}=0.05 \AA \\
\Delta \mathrm{OPtM}=0.00 \AA\end{array}$ \\
\hline $\begin{array}{l}\text { Fin de la réaction } \\
\text { End of the treatment }\end{array}$ & $\begin{array}{l}\mathrm{NPtS}=0.8 \\
\mathrm{NPtM}=5.0\end{array}$ & $\begin{aligned} \mathrm{RPtS} & =2.32 \AA \\
\mathrm{RPtM} & =2.73 \AA\end{aligned}$ & $\begin{array}{l}\Delta O \mathrm{PtS}=0.07 \AA \\
\Delta \mathrm{OPtM}=0.00 \AA\end{array}$ \\
\hline
\end{tabular}

Tableau 3: Catalyseur PtRe/Al $\mathrm{AO}_{3}-\mathrm{Cl}$ sulfuré: analyse EXAFS au seuil $\mathrm{L}_{\mathrm{II}}$ du thénium EXAFS parameters ( $\mathrm{LIII}$ thenium edge) for sulfided $\mathrm{PtRe} / \mathrm{Al}_{2} \mathrm{O}_{3}-\mathrm{Cl}$ catalyst

\begin{tabular}{|c|c|c|c|}
\hline $\mathrm{PtRe} / \mathrm{Al}_{2} \mathrm{O}_{3}-\mathrm{Cl}$ & \multicolumn{3}{|c|}{$\begin{array}{l}\text { Paramètres EXAFS obtenus au seuil } \mathrm{L}_{11} \text { du rhénium } \\
\text { EXAFS parameters obtained at the } \mathrm{L}_{\mathrm{II}} \text { edge of rhenium }\end{array}$} \\
\hline $\begin{array}{l}\text { Catalyseur sulfuré } \\
\text { initial state }\end{array}$ & $\begin{array}{r}\mathrm{NReS}=1.8 \\
\mathrm{NReM}=4.3\end{array}$ & $\begin{array}{l}\operatorname{RReS}=2.34 \AA \\
\operatorname{ReM}=2.74 \AA\end{array}$ & $\begin{aligned} \Delta O \operatorname{ReS} & =0.02 \AA \\
\Delta O \operatorname{ReM} & =0.02 \AA\end{aligned}$ \\
\hline \multicolumn{4}{|c|}{ Réduction sous hydrogène $\grave{a} 500^{\circ} \mathrm{C}$, Reduction under hydrogen at $500^{\circ} \mathrm{C}$} \\
\hline $\begin{array}{l}\text { Après } 2 h \\
\text { After } 2 h\end{array}$ & $\begin{aligned} \mathrm{NReS} & =1.8 \\
\mathrm{NReM} & =4.3\end{aligned}$ & $\begin{array}{l}\operatorname{ReS}=2.34 \AA \\
\operatorname{ReM}=2.74 \AA\end{array}$ & $\begin{aligned} \Delta \sigma \operatorname{ReS} & =0.05 \AA \\
\Delta \sigma \operatorname{ReM} & =0.05 A\end{aligned}$ \\
\hline \multicolumn{4}{|c|}{$\begin{array}{l}\text { Première réaction de reformage }\left(\mathrm{T}=500^{\circ} \mathrm{C}, \mathrm{P}\left(\mathrm{H}_{2}\right)=8 \text { bars }\right) \\
\text { First reforming reaction }\left(\mathrm{T}=500^{\circ} \mathrm{C}, \mathrm{P}\left(\mathrm{H}_{2}\right)=8 \text { bars }\right) \\
\end{array}$} \\
\hline $\begin{array}{l}\text { Après 1h } \\
\text { After } 1 \mathrm{~h}\end{array}$ & $\begin{aligned} \mathrm{N} R e S & =1.7 \\
\mathrm{NReM} & =4.0\end{aligned}$ & $\begin{array}{l}\mathrm{R} \operatorname{ReS}=2.34 \AA \\
\operatorname{ReM}=2.74 \AA\end{array}$ & $\begin{aligned} \Delta \sigma \operatorname{ReS} & =0.04 \AA \\
\Delta \sigma \operatorname{ReM} & =0.00 \AA\end{aligned}$ \\
\hline $\begin{array}{l}\text { Après } 2 \mathrm{~h} 15 \mathrm{mn} \\
\text { After } 2 \mathrm{~h} 15 \mathrm{mn}\end{array}$ & $\begin{array}{l}\mathrm{NReS}=1.8 \\
\mathrm{NReM}=4.4\end{array}$ & $\begin{array}{l}\mathrm{R} \operatorname{ReS}=2.35 \AA \\
\operatorname{ReM}=2.74 \AA\end{array}$ & $\begin{aligned} \Delta \sigma \operatorname{ReS} & =0.08 \AA \\
\Delta \sigma_{R e M} & =0.00 \AA\end{aligned}$ \\
\hline $\begin{array}{l}\text { Après } 4 \text { h10mn } \\
\text { After } 4 \text { h } 10 \mathrm{mn}\end{array}$ & $\begin{aligned} \mathrm{N} R e S & =1.8 \\
N_{R e M} & =4.3\end{aligned}$ & $\begin{array}{l}\mathrm{R} \operatorname{ReS}=2.34 \AA \\
\operatorname{ReM}=2.72 \AA\end{array}$ & $\begin{aligned} \Delta \sigma \mathrm{ReS} & =0.06 \AA \\
\Delta \mathrm{OReM} & =0.03 \AA\end{aligned}$ \\
\hline \multicolumn{4}{|c|}{$\begin{array}{l}\text { Seconde réaction de reformage }\left(\mathrm{T}=300^{\circ} \mathrm{C}, \mathrm{P}\left(\mathrm{H}_{2}\right)=3 \mathrm{bars}\right) \\
\text { Second reforming reaction }\left(\mathrm{T}=300^{\circ} \mathrm{C}, \mathrm{P}\left(\mathrm{H}_{2}\right)=3 \mathrm{bars}\right)\end{array}$} \\
\hline $\begin{array}{l}\text { Après } 30 \mathrm{mn} \\
\text { After } 30 \mathrm{mn}\end{array}$ & $\begin{array}{r}\mathrm{NReS}=1.8 \\
\mathrm{NReM}=6.1\end{array}$ & $\begin{array}{l}R_{R e S}=2.35 \AA \\
R_{R e M}=2.74 \AA\end{array}$ & $\begin{aligned} \Delta \sigma \operatorname{ReS} & =0.05 \AA \\
\Delta \sigma \operatorname{ReM} & =0.00 \AA\end{aligned}$ \\
\hline $\begin{array}{l}\text { Après 2h } \\
\text { After } 2 h\end{array}$ & $\begin{array}{r}\mathrm{NReS}=1.8 \\
\mathrm{NReM}=5.8\end{array}$ & $\begin{array}{l}\operatorname{ReS}=2.34 \AA \\
\operatorname{ReM}=2.74 \AA\end{array}$ & $\begin{aligned} \Delta \sigma \operatorname{ReS} & =0.07 \AA \\
\Delta \sigma \operatorname{ReM} & =0.00 \AA\end{aligned}$ \\
\hline $\begin{array}{l}\text { Fin de la réaction } \\
\text { End of the treatment }\end{array}$ & $\begin{aligned} \mathrm{N}_{\mathrm{ReS}} & =1.8 \\
\mathrm{~N} \mathrm{ReM} & =5.6\end{aligned}$ & $\begin{array}{l}R_{R e S}=2.35 \AA \\
\operatorname{ReM}=2.73 \AA\end{array}$ & $\begin{aligned} \Delta \sigma_{\operatorname{ReS}} & =0.04 \AA \\
\Delta \mathrm{O}_{\mathrm{ReM}} & =0.00 \AA\end{aligned}$ \\
\hline
\end{tabular}




\section{L'ACTIVITE CATALYTIQUE DU MONOMETALLIQUE PU/Al $\mathrm{O}_{3}$-Cl ET DU BIMETALLIQUE PtRe/AI $\mathrm{O}_{3}$-Cl SULFURES LORS DE LA REACTION DE REFORMAGE DU n-HEPTANE}

Durant la réaction de reformage et à différents moments, nous avons effectué des analyses chromatographiques pour suivre l'évolution de l'activité de nos catalyseurs (le monométallique $\mathrm{Pt} / \mathrm{Al}_{2} \mathrm{O}_{3}-\mathrm{Cl}$ et le bimétallique $\mathrm{PtRe} / \mathrm{Al}_{2} \mathrm{O}_{3}-\mathrm{Cl}$ sulfurés). Les principaux constituants obtenus sont des iso-alcanes, des alcènes, des aromatiques et aussi des produits issus du crackage. L'activité de nos catalyseurs est exprimée par le taux de conversion de notre réactif de départ n-heptane et qui représente le pourcentage du n-heptane transformé (tableaux 4a et 4b).

Tableau 4a: Evolution de l'activité catalytique (taux total de conversion du $n$-heptane $=\tau$ ) pour les deux catalyseurs sulfurés, le monométallique $\mathrm{Pt} / \mathrm{Al}_{2} \mathrm{O}_{3}-\mathrm{Cl}$ et le bimétallique $\mathrm{PtRe} / \mathrm{Al}_{2} \mathrm{O}_{3}-\mathrm{Cl}$ durant la première reaction de reformage.

Catalytic activity evolution (total rate conversion of $n$-heptane $=\tau$ ) for sulfided $\mathrm{Pt} / \mathrm{Al}_{2} \mathrm{O}_{3}-\mathrm{Cl}$ and $\mathrm{PtRe} / \mathrm{Al}_{2} \mathrm{O}_{3}-\mathrm{Cl}$ catalysts during the first reforming reaction.

\begin{tabular}{|c|c|c|c|}
\hline \multicolumn{4}{|c|}{$\begin{array}{c}\text { Première réaction de reformage }\left(\mathrm{T}=500^{\circ} \mathrm{C}, \mathrm{P}\left(\mathrm{H}_{2}\right)=8 \text { bars }\right) \\
\text { First reforming reaction }\left(\mathrm{T}=500^{\circ} \mathrm{C}, \mathrm{P}\left(\mathrm{H}_{2}\right)=8 \text { bars }\right)\end{array}$} \\
\hline \multicolumn{2}{|c|}{$\mathrm{Pt} / \mathrm{Al}_{2} \mathrm{O}_{3}-\mathrm{Cl}$} & \multicolumn{2}{c|}{ PtRe/Al $\mathrm{O}_{3}$-Cl } \\
\hline $\begin{array}{c}\text { Après 1h45mn } \\
\text { After 1h45mn }\end{array}$ & $\tau=55 \%$ & $\begin{array}{l}\text { Après } 1 \mathrm{~h} 30 \mathrm{mn} \\
\text { After } 1 \mathrm{~h} 30 \mathrm{mn}\end{array}$ & $\tau=78 \%$ \\
\hline $\begin{array}{c}\text { Après 3h30mn } \\
\text { After 3h30mn }\end{array}$ & $\tau=50 \%$ & $\begin{array}{l}\text { Après } 3 \mathrm{~h} 40 \mathrm{mn} \\
\text { After 3h40mn }\end{array}$ & $\tau=70 \%$ \\
\hline & $\begin{array}{l}\text { Après } 5 \mathrm{~h} 30 \mathrm{mn} \\
\text { After } 5 \mathrm{~h} 30 \mathrm{mn}\end{array}$ & $\tau=65 \%$ \\
\hline
\end{tabular}

Tableau 4b: Evolution de lactivite catalytique (taux total de conversion du n-heptane $=\tau$ ) pour les deux catalyseurs sulfurés, le monométallique $\mathrm{Pt} / \mathrm{Al}_{2} \mathrm{O}_{3}-\mathrm{Cl}$ et le bimétallique $\mathrm{PtRe} / \mathrm{Al}_{2} \mathrm{O}_{3}-\mathrm{Cl}$ durant la seconde reaction de reformage.

Catalytic activity evolution (total rate conversion of $n$-heptane $=\tau$ ) for sulfided $\mathrm{Pt} / \mathrm{Al}_{2} \mathrm{O}_{3}-\mathrm{Cl}$ and $\mathrm{PtRe} / \mathrm{Al}_{2} \mathrm{O}_{3}-\mathrm{Cl}$ catalysts during the second reforming reaction.

\begin{tabular}{|c|c|c|c|}
\hline \multicolumn{3}{|c|}{$\begin{array}{c}\text { Seconde réaction de reformage }\left(\mathrm{T}=300^{\circ} \mathrm{C}, \mathrm{P}\left(\mathrm{H}_{2}\right)=3 \text { bars }\right) \\
\text { Seconde reforming reaction }\left(\mathrm{T}=300^{\circ} \mathrm{C}, \mathrm{P}\left(\mathrm{H}_{2}\right)=3 \text { bars }\right)\end{array}$} \\
\hline \multicolumn{3}{|c|}{$\mathrm{Pt} / \mathrm{Al}_{2} \mathrm{O}_{3}-\mathrm{Cl}$} & \multicolumn{1}{c|}{$\mathrm{Pt}-\mathrm{Re} / \mathrm{Al} \mathrm{O}_{3}-\mathrm{Cl}$} \\
\hline \multirow{2}{\text{Après}1\mathrm{h}}{} & $\tau=1,2 \%$ & $\begin{array}{c}\text { Après } 1 \mathrm{~h} 30 \mathrm{mn} \\
\text { After } 1 \mathrm{~h} 30 \mathrm{mn}\end{array}$ & $\tau=0.35 \%$ \\
After $1 \mathrm{~h}$ & & & $\tau$ \\
\hline
\end{tabular}

\section{DISCUSSION}

La deuxième partie de notre investigation, dont le but était la corrélation possible entre la structure de la phase métallique de nos deux catalyseurs $\left(\mathrm{Pt} / \mathrm{Al}_{2} \mathrm{O}_{3}-\mathrm{Cl}\right.$ et $\mathrm{PtRe} / \mathrm{Al}_{2} \mathrm{O}_{3}-\mathrm{Cl}$ sulfurés) et leurs activités catalytiques, permet de voir une différence de cette activité au début de la réaction de reformage $\left(\mathrm{T}=500^{\circ} \mathrm{C}, \mathrm{P}\left(\mathrm{H}_{2}\right)=8\right.$ bars $)$. Le bimétallique $\mathrm{PtRe} / \mathrm{Al}_{2} \mathrm{O}_{3}-\mathrm{Cl}$ se révèle beaucoup plus actif que le monométallique $\mathrm{Pt}_{\mathrm{Al}} \mathrm{O}_{3}-\mathrm{Cl}$.

En examinant les informations structurales obtenues, on remarque que la phase métallique de l'échantillon $\mathrm{Pt} / \mathrm{Al}_{2} \mathrm{O}_{3}-\mathrm{Cl}$ sulfuré, après la réduction à $500^{\circ} \mathrm{C}$ et avant le début de la réaction de reformage, est reconstruite. On passe donc d'un monométallique sulfuré, où au départ les atomes de platine sont entourés essentiellement par des atomes de soufre, à un catalyseur présentant une phase métallique libérée de ces voisins soufre. Par contre, le nombre assez grand de voisins métalliques autour du platine indique un accroissement de la taille des agrégats métalliques et donc une diminution de la dispersion de la phase active sur le support.

La phase métallique du système $\mathrm{PtRe} / \mathrm{Al}_{2} \mathrm{O}_{3}-\mathrm{Cl}$, quant à elle, ne subit pas de changements significatifs. Ces informations structurales nous aident alors à comprendre et à expliquer la différence d'activité catalytique qui existe entre les deux systèmes et donc de trouver une corrélation entre la structure et l'activité de chaque système. 
Si la régénération de la phase métallique du système $\mathrm{P} / \mathrm{Al}_{2} \mathrm{O}_{3}-\mathrm{Cl}$ sulfuré, après la réduction à $500^{\circ} \mathrm{C}$, explique son activité non négligeable de départ (de l'ordre de $50 \%$ ), l'accroissement de la taille de ses agrégats métalliques explique le fait que cette activité reste bien inférieure à celle du système bimétallique $\mathrm{PtRe} / \mathrm{Al}_{2} \mathrm{O}_{3}-\mathrm{Cl}$ (de l'ordre de $80 \%$ ). La phase métallique de ce dernier étant restée stable après la reduction sous hydrogène à $500^{\circ} \mathrm{C}$, et ne subissant donc pas de frittage, laisse ainsi une accessibilité plus grande aux sites métalliques actifs comparé au monométallique $\mathrm{Pt} / \mathrm{Al}_{2} \mathrm{O}_{3}-\mathrm{Cl}$. Dans une étude récente, Vaarkamp et al. [6] montrent que la sulfuration d'un catalyseur monométallique platine supporté sur zeolithe (Pt/BaK-LTD) provoque un accroissement de la taille de ses agrégats métalliques, conduisant ainsi à un empoisonnement du catalyseur.

Après la première étape de réaction de reformage $\left(\mathrm{T}=500^{\circ} \mathrm{C}, \mathrm{P}\left(\mathrm{H}_{2}\right)=8 \mathrm{bars}\right)$, la deuxième étape de cette réaction réalisée dans des conditions plus sévères $\left(T=300^{\circ} \mathrm{C}, P\left(\mathrm{H}_{2}\right)=3 \mathrm{bars}\right)$ montre que les deux catalyseurs sont pratiquement inactifs dans ces conditions (le taux de conversion de n-heptane est pratiquement nul). Le passage de la première étape de reformage à la deuxième s'accompagne toutefois d'un accroissement apparemment faible de la taille des agrégats métalliques, et ce pour les deux systèmes catalytiques. Cette diminution de la dispersion de leurs phases métalliques ne peut expliquer à elle seule la chute brutale de leur activité. En effet, les conditions de la deuxième étape de la réaction de reformage $\left(\mathrm{T}=300^{\circ} \mathrm{C}\right.$, $\mathrm{P}\left(\mathrm{H}_{2}\right)=3$ bars) favorisent le dépôt de coke. La perte d'activité dans ces conditions est donc attribuée principalement à l'empoisonnement de la phase active par ce dépôt.

On citera ici en référence une étude de N.S. Guyot-Sionnest et al. $[7,8]$ qui a montré que le système monométallique $\mathrm{Pt} / \mathrm{Al}_{2} \mathrm{O}_{3}-\mathrm{Cl}$ est pratiquement inactif pour une réaction de reformage du n-heptane réalisée dans des conditions proches de celles utilisées lors de la deuxième étape de notre réaction. Cette inactivité a été clairement attribuée à l'empoisonnement de la phase métallique par un dépôt de carbone visible. Les mêmes conclusions ont été tirées d'une étude récente d'A. Caballero et al. [9] sur un catalyseur bimétallique $\mathrm{PtRe} / \mathrm{Al}{ }_{2} \mathrm{O}_{3}-\mathrm{Cl}$ où des voisins carbone autour des éléments métalliques ont été, là encore, détectés. Si, dans notre cas, nous n'avons pu identifier de voisins carbone autour des éléments métalliques, cela peut s'expliquer par le fait que :

- pour le catalyseur $\mathrm{Pt} / \mathrm{Al}_{2} \mathrm{O}_{3}-\mathrm{Cl}$ sulfuré, à la fin de la réaction de reformage, la relativement importante taille des agrégats métalliques formés empêche cette détection, le signal carbone étant certainement trop faible;

- pour le système $\mathrm{PtRe} / \mathrm{Al}_{2} \mathrm{O}_{3}-\mathrm{Cl}$ sulfuré, en plus de l'accroissement de la taille des agrégats métalliques, l'existence de voisins soufre en nombre important autour des éléments métalliques (platine et thénium) rend la détection de voisins carbone encore plus difficile.

Ces interactions agrégats métalliques-dépôt de coke n'ont donc pu être directement mises en évidence. Nous croyons néanmoins que la raison essentielle de la perte d'activité est la même, c'est-à-dire un empoisonnement direct.

\section{CONCLUSION}

Ce travail confirme tout d'abord l'importance des mesures EXAFS faites en conditions réactionnelles pour apporter des informations structurales en liaison avec la réactivité des systèmes catalytiques de reformage. Nous avons pu en effet apporter des informations claires sur la différence de comportement entre le catalyseur monométallique $\mathrm{Pt} / \mathrm{Al}_{2} \mathrm{O}_{3}-\mathrm{Cl}$ et le catalyseur bimétallique PtRe/ $\mathrm{Al}_{2} \mathrm{O}_{3}-\mathrm{Cl}$, notamment lors de l'étape de sulfuration. L'utilisation du dispositif instrumental de traitement haute temperature - haute pression nous a permis, dans ce travail, de procéder au même type dinvestigation pour la mise en régime de ces matériaux.

L'étude dans les conditions de reformage menée sur ces deux systèmes sulfurés nous a conduit à montrer en quoi le bimétallique $\mathrm{PtRe} / \mathrm{Al}_{2} \mathrm{O}_{3}-\mathrm{Cl}$ sulfuré présente le meilleur intérêt catalytique. Sa phase métallique est en effet connue pour être plus résistante par rapport à l'espèce monométallique $\mathrm{Pt} / \mathrm{Al}_{2} \mathrm{O}_{3}-\mathrm{Cl}$ sulfuré. Le résultat de nos expériences d'absorption $\mathrm{X}$ in situ indique que la phase métallique de ce dernier subit une agglomération dès la réduction sous hydrogène a $500^{\circ} \mathrm{C}$, processus qui s'accentue jusqu'à la fin de la réaction de reformage.

A contrario, la phase métallique associée au bimétallique ne subit cette agglomération qu'a partir de la deuxième partie de la réaction de reformage. On a alors complété ces études structurales par des mesures d'activité catalytique qui confirment que le catalyseur bimétallique sulfuré est beaucoup plus actif que le monométallique sulfuré. On a donc ici un 
ensemble cohérent de résultats qui nous permet de bien comprendre le comportement de nos deux systèmes catalytiques.

\section{Références}

[1] Prins R. and Koningsberger D. C., in "X-Ray Absorption : Principles, Applications, Techniques of EXAFS, SEXAFS and XANES", Ed. J. Wiley \& Sons, (1987).

[2] Shum V. K., Butt J. B., Sachtler W. M., J. Cat. 96 (1985) 371.

[3] Parera J. M., Beltramini J. N., Querini C. A., Martinelli E. E., Churin E. J., Aloe P. E., Figoli N. S., J. Cat. 99 (1986) 39.

[4] Bensaddik A., Dexpert H., Bazin D., Caballero A., Villain F., Lynch J., Didillon B., Physica B., 208\&209 (1995) 677.

[5] Kluksdahl H. E., U.S. Pat., 3415737 (1968).

[6] Vaarkamp M., Miller J. T., Modica F. S., Lane G. S., Koningsberger D. C., J. Cat. 138 (1992) 675.

[7] Guyot-Sionnest N. S., Villain F., Bazin D., Dexpert H., LePeltier F., Lynch J., Bournonville J. P., Catal. Lett., 8 (1991) 283.

[8] Guyot-Sionnest N. S., Villain F., Bazin D., Dexpert H., LePeltier F., Lynch J., Catal. Lett., 8 (1991) 297.

[9] Caballero A., Dexpert H., Villain F., LePeltier F., Lynch J., J. Chem. Soc. Faraday Trans., 89(1) (1993) 159. 